\title{
INMUNOMODULACIÓN DE Uncaria tomentosa SOBRE CÉLULAS DENDRÍTICAS, IL-12 Y PERFIL TH1/TH2/TH17 EN CÁNCER DE MAMA
}

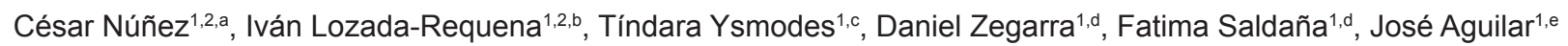

\author{
RESUMEN
}

Objetivos. Evaluar el efecto inmunomodulador del extracto estandarizado (5,03\%, alcaloides oxindólicos pentacíclicos) de Uncaria tomentosa (UT-POA) sobre el inmunofenotipo de células dendríticas (DC) y IL-12/Th1/Th2/Th17 en pacientes con cáncer de mama estadio II (BCII) y mujeres sanas $(\mathrm{H})$. Materiales y métodos. Se obtuvó sangre de $11 \mathrm{H}$ y $7 \mathrm{BCIl}$, se aislaron y cultivaron PBMC por $2 \mathrm{~h}$ con/sin distintas concentraciones de UT-POA y se estimularon o no con LPS por $24 \mathrm{~h}$. Las PBMC fueron marcadas con anticuerpos específicos para DC y en el sobrenadante se midió las citoquinas Th1/Th2/Th17, ambos por citometría de flujo. Además, se midió IL-12 por ELISA. Resultados. UT-POA no alteró a las DC o la expresión de moléculas accesorias en BCll. Sin embargo, en H mostró una disminución en el porcentaje de DC mieloides $(\mathrm{mDC})$ con incremento de HLA-DR y CD86 a $1000 \mu \mathrm{g} / \mathrm{mL}$. La secreción de IL-12 fue modificada solo en $\mathrm{H}$, incrementando la subunidad p70 y disminuyendo la p40. UT-POA incremento Th1 (IFN-y y IL-2), Th2 (IL-4) y Th17 (IL-17) en BCIl y H. Conclusiones. UT-POA incrementa la producción de citoquinas relacionadas con la respuesta antitumoral a concentraciones entre el rango de $500-1000 \mu \mathrm{g} / \mathrm{mL}$. Este efecto positivo debería ser evaluado no solo sistemicamente sino también en el microambiente tumoral. La UT-POA puede ser un fitoquímico útil en la quimioprevención y en el uso alternativo con terapias contra el cáncer.

Palabras clave: Uncaria tomentosa; Células dendríticas; IL-12; Cáncer de mama (fuente: DeCS BIREME).

\section{IMMUNOMODULATION OF Uncaria tomentosa OVER DENDRITIC CELLS, IL-12 AND PROFILE TH1/TH2/TH17 IN BREAST CANCER}

\begin{abstract}
Objetives. This study aimed to research the in vitro immunomodulatory effects of an Uncaria tomentosa hydroalcoholic extract standardized (5.03\%, pentacyclic oxindole alkaloids) (UT-POA) on the immunophenotype of dendritic cells (DC) subsets, Th1, Th2, Th17 and IL-12 cytokines from patients with stage II breast cancer (BCII) and healthy women (H). Materials and methods. Blood of $11 \mathrm{H}$ and $7 \mathrm{BCll}$ was obtained, PBMC were isolated and cultured for $2 \mathrm{~h}$ with/without various concentrations of UT-POA and stimulated or not with LPS for $24 \mathrm{~h}$. PBMC were labeled with specific antibodies for DC and in the supernatant we measured Th1/Th2/Th17 cytokines, both by flow cytometry. Furthermore IL-12 was measured by ELISA. Results. UT-POA did not alter DC or accessory molecules expression in BClI. However, H exhibited a decrease in the percentage of $\mathrm{mDC}$ (myeloid DC) and an increase in HLA-DR and CD86 expression at $1000 \mu \mathrm{g} /$ $\mathrm{mL}$. IL-12 secretion was modified only in the $\mathrm{H}$ group, increasing p70 subunit and decreasing p40 subunit. UT-POA increased Th1 (IFN-y and IL-2), Th2 (IL-4) and Th17 (IL-17) secretion in both groups. Conclusions. UT-POA increased the production of cytokines related with anti-tumoral response at concentrations of $500-1000 \mu \mathrm{g} / \mathrm{mL}$. This positive effect should be evaluated not only systemically but also in the tumor microenvironment in further studies. UT-POA may be a useful phytochemical in chemoprevention and in the alternative use in cancer therapies.
\end{abstract}

Key words: Uncaria tomentosa; Dendritic cells; IL-12; Breast cancer (source: MeSH NLM).

\section{INTRODUCCIÓN}

La Uncaria tomentosa (Willd.) DC (Rubiaceae) (UT) o uña de gato, es una liana de la selva peruana y de zonas tropicales de América del Sur y Central. Grupos indígenas como los asháninkas utilizaban esta planta para mejorar su salud (1). La UT es usada como anticonceptivo, anticancerígeno, en desordenes inflamatorios y gastrointestinales (2). Es consumida como infusión ${ }^{(3)}$. Sandoval-Chacón et al., y Aguilar et al., demostraron su actividad antinflamatoria in vitro ${ }^{(4,5)}$. Aquino et al. lo hizó in vivo con modelos de inflamación

\footnotetext{
1 Laboratorio de Inmunología. Departamento de Ciencias Celulares y Moleculares. Facultad de Ciencias y Filosofía. Universidad Peruana Cayetano Heredia. Lima, Peru.

2 EMINDES SAC (Empresa de Investigación y Desarrollo en Cáncer). Lima, Peru.

a Médico general; ${ }^{\mathrm{b}}$ magíster en Ciencias; ${ }^{\mathrm{c}}$ licenciada en Ciencias; ${ }^{\mathrm{d}}$ bachiller en Ciencias; ${ }^{\mathrm{e}}$ médico reumatólogo, especialista en Inmunología Recibido: : 09-02-15 Aprobado: 08-07-15
} 
en ratones (2). Dreifuss et al.demostró sus efectos antitumorales y antioxidantes en un modelo in vivo de carcinosarcoma en ratas ${ }^{(6,7)}$.

El rol de la inflamación crónica en la iniciación y progression del tumor es conocido, por lo tanto, la conexión entre inflamación y cáncer no es de sorprender ${ }^{(8,9)}$. La UT ha demostrado inhibir la translocación del factor de transcripción NF-kB ${ }^{(4,5)}$, un factor que activa genes involucrados con la respuesta antinflamatoria y con la regulación de apoptosis. En células normales la activación de NF-kB promueve la apoptosis, pero en células malignas promueve la supervivencia, mientras que su inhibición puede disminuir el crecimiento tumoral ${ }^{(10)}$. AllenHall L et al., demostró que el tratamiento con UT inhibió la activación dependiente de LPS de componentes específicos de la vía de señalización AP-1 ${ }^{(11)}$. Una efectiva respuesta antitumoral requiere la participación activa de células presentadoras de antígenos (CPA) responsables de la presentación de antígenos tumorales ${ }^{(12)}$. La importancia de las CPA recae en el hecho de que defectos en la función de los linfocitos que infiltran el tumor en pacientes con cáncer y linfocitos T (LT) de ratones portadores de tumor puede ser completamente revertida cuando una efectiva presentación de antígeno tiene lugar y se provee de IL-2 exógena a estos linfocitos. Las células dendríticas (DC) son las más potentes CPA ${ }^{(13,14)}$. Las DC tienen un rol central en la inmunidad antitumoral debido a que procesan antígenos tumorales y estimulan a LT antígenoespecíficos $(15,16)$. Para la efectiva presentación de antígenos es importante una óptima señal coestimuladora entre ambas células ${ }^{(12)}$. Una población de DC aisladas de sangre periférica de pacientes con cáncer de mama y de cabeza y cuello exhibió una reducida capacidad de agruparse y de estimular alogénicamente respuestas por LT antígeno-específicos. Las DC aisladas de estos pacientes expresaban un bajo nivel de expresión de $\mathrm{MHC}$ II (HLA-DR) y moléculas coestimuladoras que las DC de donadores sanos ${ }^{(17)}$. Nuestro equipo ha demostrado que un extracto hidroalcohólico de UT disminuyó el porcentaje de DC mieloides (DCm) e incrementó el HLA-DR y el CD86 de manera dosis dependiente en células mononucleares de sangre periférica (PBMC) de pacientes con artritis reumatoide, sugiriendo un efecto inmunomodulador ${ }^{(18)}$.

El nuevo paradigma del balance immune no solo involucra a los LT citotóxicos CD8, subpoblaciones de LT CD4, linfocitos reguladores, linfocitos gamma-delta, Natural killer, Natural killer T cells, macrofágos, DC y otras células inmunes innatas, sino también a citoquinas inflamatorias, Th1, Th2, Th17 e inmunoreguladoras. El IL-12 favorece el perfil Th1, y Th1 es una de las más efectivas respuestas contra los tumores. Los macrófagos y DC participan en la respuesta antitumoral produciendo IL-12 entre otros roles propios de la inmunidad innata. En un estudio in vitro encontramos que la UT incrementaba los niveles de IL-12p70 en PBMC de sujetos sanos (datos no publicados), lo que suguiere que la UT promovería una respuesta Th1. Domingues $A$. et al., demostró en ratones Balb-c sanos que la UT promovía un perfil Th2 (IL-4, IL-5) de manera dosis dependiente, mientras que inhibía el perfil Th1 (IL-2, TNF- $\alpha$, IFN-k) ${ }^{(19)}$. Fazio AL et al., en macrófagos peritoneales de ratones C57/BL6, portadores de melanoma B16/BL6, demostró que la UT inhibía IL-6 y NO pero no TNF-a ${ }^{(20)}$. Urdanibia et al., encontraron un efecto antinflamatorio y antitumoral en otra especie de Uncaria, la $U$. guianensis, al observar la inhibición del crecimiento del tumor mamario (4T1) relacionado probablemente con la disminución de mediadores inflamatorios como TNF-a e IL-6 a través de la inhibición de la vía NFkB ${ }^{(21)}$. En este estudio reportamos la actividad antitumoral in vitro de un extracto hidroalcohólico de UT así como también un efecto inmunomodulador sobre DC y las citoquinas IL12, Th1, Th2, Th17 a partir de PBMC humanos.

\section{MATERIALES Y MÉTODOS}

\section{PERSONAS}

Participaron diez mujeres sanas con mamografía negativa para adenocarcinoma mamario (46,6 \pm 9,1 años) (H) y siete mujeres histológicamente diagnósticadas con adenocarcinoma mamario en estadio II (54,7 \pm 12,7 años) (BCII). Los criterios de inclusión fueron, no haber sido: tratada con ningún ciclo de quimio/radioterapía; sometida a cirugía; tenido otro cáncer; sufrido de inmunodeficienciaz y/o enfermedades autoinmunes; recibido citostáticos en los últimos 12 meses y paciente con enfermedad crónica. Se obtuvieron por venipuntura muestras de sangre periférica $(36 \mathrm{~mL})$.

\section{EXTRACTO HIDROALCOHÓLICO DE UT ESTANDARIZADO AL 5,03\% DE ALCALOIDES OXINDÓLICOS PENTACÍCLICOS (UT-POA)}

El extracto fue proporcionado por Peruvian Heritage S.A.C. Se preparó un extracto hidroalcohólico de la corteza de $U$. tomentosa, por decocción con etanol y agua en la proporción 7:3 durante $1 \mathrm{~h}$ a $20{ }^{\circ} \mathrm{C}$ y desecado por atomización cuyo resultado fue un polvo. El contenido de alcaloides oxindólicos pentacíclicos fue $5,03 \%$, verificado por cromatografía líquida de alta performance (HPLC) de acuerdo con Dreifuss AA et al. Se preparó una solución stock de UT-POA diluyendo $30 \mathrm{~g} / \mathrm{L}$ de agua bidestilada la cual fue hervida por 30 min. Luego de reposar, se decantó la solución clara de los restos sólidos y se filtró dos veces en Whatman N. ${ }^{\circ} 3$ y se microfiltró $(0,22 \mu \mathrm{m})$, la solución stock fue almacenada a $-70^{\circ} \mathrm{C}$. 


\section{AISLAMIENTO DE PBMC}

Los PBMC fueron obtenidos por centrifugación en gradiente de densidad con Histopaque 1,077 g/ $\mathrm{mL}$ (Sigma, St Louis, MO, USA). Previamente, se prepararon tubos con $10 \mathrm{~mL}$ de Histopaque. Las muestras de sangre fueron diluidas 1:1 con RPMI1640 completo (Sigma, St Louis, MO, USA). La sangre diluida $(30 \mathrm{~mL})$ fue agregada sobre el Histopaque $y$ centrifugada a $400 \mathrm{~g}$ por $30 \mathrm{~min}$. Los PBMC fueron colectados y almacenados en hielo.

\section{CULTIVO CELULAR Y GRUPOS DE ENSAYO}

Los PBMC fueron cultivados en medio RPMI-1640 con $10 \%$ de suero fetal bovino (Hyclone), penicilina $100 \mathrm{U} / \mathrm{mL}$ y estreptomicina $100 \mu \mathrm{g} / \mathrm{mL}$ (Sigma, St Louis, MO, USA). $1 \times 10^{6}$ PBMC fueron colocados en tubos de citometría e incubados a $37{ }^{\circ} \mathrm{C}$ en $5 \% \mathrm{CO}_{2}$ por $2 \mathrm{~h}$ con/sin UT-POA, se agregó o no lipopolisacáridos de E. coli (Sigma, St Louis, MO, USA) (LPS $1 \mathrm{ug} / \mathrm{mL}$ ), estandarizados en nuestro laboratorio y se incubaron a $37^{\circ} \mathrm{C}$ en $5 \% \mathrm{CO}_{2}$ durante $24 \mathrm{~h}$. Los grupos de ensayo para las pacientes BCII y $\mathrm{H}$ fueron: $\mathrm{A}$. Basal = $\mathrm{PBMC}$ más vehículo de UTPOA; $B$. LPS = PBMC más vehículo y estimulados con LPS $(1 \mu \mathrm{g} / \mathrm{mL}) ; C$. UG50 = PBMC más UT-POA $(50 \mu \mathrm{g} / \mathrm{mL})$ y estimulados con LPS; D. UG500 = PBMC más UT-POA $(500 \mu \mathrm{g} / \mathrm{mL})$ y estimulados con LPS y E. UG1000 = PBMC más UT-POA $(1000 \mu \mathrm{g} /$ $\mathrm{mL}$ ) y estimulados con LPS.

\section{CITOMETRÍA DE FLUJO PARA DC Y HLA-DR/CD86}

Antes de la citometría de flujo, los sobrenadantes de cultivo fueron separados para la medición de citoquinas, y las células fueron lavadas y resuspendidas en solución de lavado (Cell wash solution, $1 \%$ suero bovino fetal en PBS $(\mathrm{pH} 7,4))$. Inmediatamente, las células fueron marcadas con anticuerpos específicos: anti-Lin1-FITC, anti-HLA-DR-PerCP, anti-CD11C-APC/anti-CD123APC y anti-CD86-PE (Becton Dickinson, San Jose, CA, USA). Las células marcadas fueron incubadas por 30 minutos a $2-8{ }^{\circ} \mathrm{C}$, y lavadas dos veces con $1 \mathrm{~mL}$ de cell wash a $400 \mathrm{~g}$ por $5 \mathrm{~min}$.

Las células fueron resuspendidas en $500 \mu \mathrm{L}$ de Cell fix (paraformaldehido al $1 \%$ en PBS pH 7,4 ) y almacenadas a 4 ${ }^{\circ} \mathrm{C}$ hasta su lectura. La adquisición fue hecha en un citómetro de flujo FACSCanto ${ }^{\mathrm{TM}}$ II (BD Immunocytometry Systems, USA). El análisis de datos se realizó con el software Summit 4.3 (Dako Colorado, Inc., USA). Se determinaron porcentajes de DC mieloides (DCm) (CD11C+HLA-DR+Lin-1-) y plasmacitoides (DCp) (CD123+HLA-DR+Lin-1-). En cada subpoblación se midió la intensidad de fluorescencia media (IFM) de HLA-DR y CD86.
MEDICIÓN DE CITOQUINAS TH1/TH2/TH17 POR CYTOMETRIC BEAD ARRAY (CBA)

Se usó un kit de CBA para la cuantificación de Th1/ Th2/Th17 (BD, San José CA, USA) en $50 \mu \mathrm{L}$ del sobrenadante de cultivo celular. Se midieron: interferongamma (IFN-k), interleuquina-2 (IL-2), factor de necrosis tumoral tipo alpha (TNF- $\alpha$ ), interleuquina-4 (IL-4), interleuquina-6 (IL-6), interleuquina-10 (IL-10) e interleuquina-17A (IL-17A). Se sigueron estrictamente las recomendaciones del fabricante. La adquisición de datos se hizo en un citómetro de flujo FACSCanto $^{\text {TM }}$ II (BD Immunocytometry Systems, USA) y el análisis con el software $\mathrm{BD}^{\mathrm{TM}}$ Cytometric Bead Array Software, version 1.4 .

MEDICIÓN DE IL-12 Y IL-6 POR ENZIMAINMUNOENSAYO (ELISA)

Se usaron kits de ELISA (EIAOpt BDBiosciences, San Diego, CA, USA) para IL-12p40, IL-12p70 y IL-6. Se siguió estrictamente las recomendaciones del fabricante. Los niveles de IL- 6 medidos por CBA estuvieron por encima del límite superior de detección (5000 pg/ $\mathrm{mL}$ ) por esta razón fue necesario diluir las muestras y medirla por ELISA.

\section{ANÁLISIS ESTADÍSTICO}

Los resultados se expresan como el promedio \pm error estándar de la media. Se realizaron las pruebas no paramétricas de Kruskal-Wallis seguido por la prueba de comparaciones multiples de Dunn y test $\mathrm{t}$ seguido por el test de comparaciones de rangos de Mann Whitney (twotailed) usando GraphPad Prism versión 6.00 para Mac, GraphPad Software, La Jolla California USA. Valores de $p<0,05$ fueron considerados estadísticamente significativos.

\section{CONSIDERACIONES ÉTICAS}

El Comité Institucional de Ética Humana de la Universidad Peruana Cayetano Heredia aprobó el presente estudio (código de registro 57825) y cada participante firmó un consentimiento informado

\section{RESULTADOS}

\section{$D C m, D C p$ y $H L A-D R / C D 86$}

La medición de DCm, DCp y la expresión de HLADR y CD86 se hizó con $1 \times 10^{6}$ PBMC. Después del tratamiento con UT-POA se encontró una disminución dosis-dependiente del \%DCm en el grupo $\mathrm{H}$, la que fue significativa a $500 \mu \mathrm{g} / \mathrm{mL}$ y $1000 \mu \mathrm{g} / \mathrm{mL}(\mathrm{p}<0,05)$ 

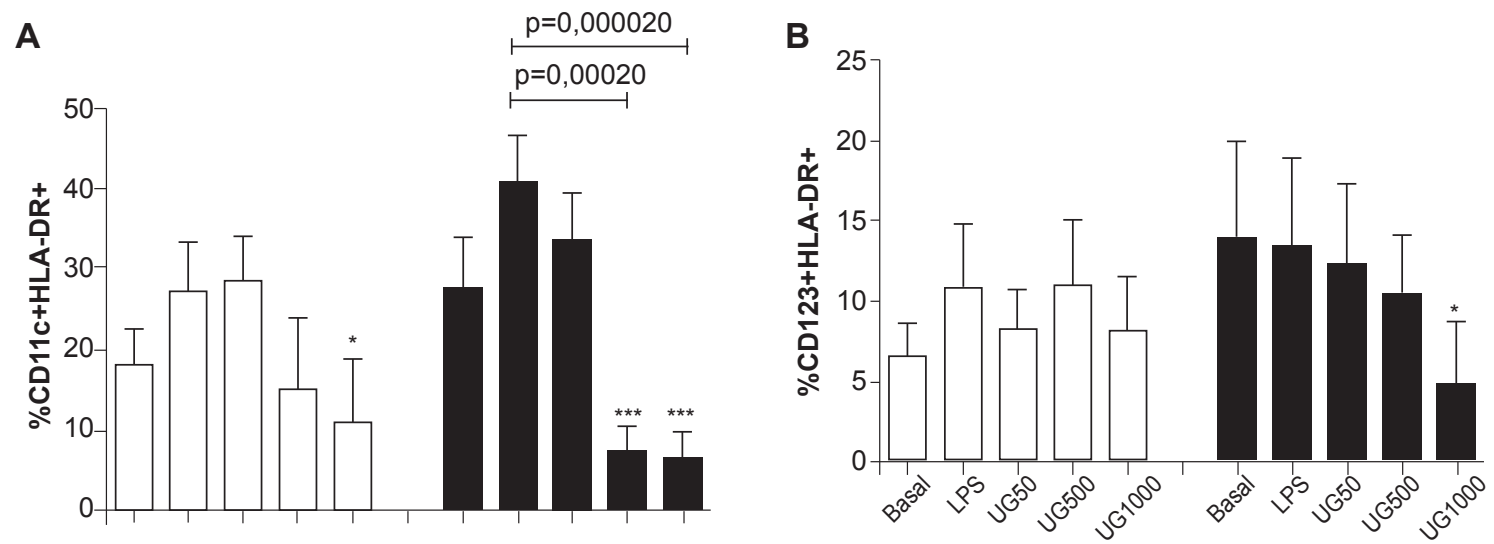

Figura 1. Efecto de UT-POA sobre el porcentaje de A) DCm (CD11c+HLA-DR+) y B) DCp (CD123+HLA-DR+). Se cultivaron 1x106 PBMC/tubo y se trataron por $2 \mathrm{~h}$ con UT-POA $(50,500$ y $1000 \mu \mathrm{g} / \mathrm{mL})$ excepto los grupos Basal y LPS, los que recibieron el vehículo. Luego, todos excepto el Basal fueron estimulados con LPS $(1 \mu \mathrm{g} / \mathrm{mL})$ por $24 \mathrm{~h}$. Finalmente, las células fueron marcadas con anticuerpos específicos para DC y medidas por citometría de flujo. Los datos fueron adquiridos y los porcentajes de las poblaciones celulares fueron analizados con Summit 4.3. Los datos representan el promedio \pm error estándar de la media de $7 \mathrm{BCll}$ y $10 \mathrm{H}$. Se realizaron el test de Kruskal-Wallis seguido por el test de comparaciones multiples de Dunn y test t seguido por el test de Mann Whitney (two-tailed) (GraphPad Prism v 6.00) Valores de $p<0,05$ con respecto a LPS fueron considerados significativos. BCIl=pacientes con cáncer de mama estadio II; $\mathrm{H}=$ mujeres sanas con mamografías negativas; UT-POA= extracto hidroalcohólico de Uncaria tomentosa con $5,03 \%$ de alcaloides oxindólicos pentacíclicos; $\mathrm{DCm}=$ células dendríticas mieloides; $\mathrm{DC} p=$ células dendríticas plasmacitoides y $\mathrm{PBMC}=$ células mononucleares de sangre periférica; ${ }^{*} p<0,05 ;{ }^{* * *} p<0,001$.

Figura 2. Efecto de UT-POA sobre la expresión de moléculas de maduración y activación de DC. Se cultivaron $1 \times 10^{6}$ PBMC/tubo y se trataron por $2 \mathrm{~h}$ con UTPOA (50, 500 y $1000 \mu \mathrm{g} / \mathrm{mL}$ ) excepto los grupos Basal y LPS, los que recibieron el vehículo. Luego, todos excepto el Basal fueron estimulados con LPS $(1 \mu \mathrm{g} / \mathrm{mL})$ por $24 \mathrm{~h}$. Finalmente, las células fueron marcadas con anticuerpos específicos para las moléculas HLA-DR y CD86 y medidas por citometría de flujo. Los datos fueron adquiridos y la IFM de las moléculas fueron analizadas con Summit 4.3. A) nivel de expresión de HLA-DR en DCm; B) nivel de expresión de CD86 en DCm; C) nivel de expresión de HLA-DR en DCp; B) nivel de expresión de CD86 en DCp. Los datos representan el promedio \pm error estándar de la media de $7 \mathrm{BCll}$ y $10 \mathrm{H}$. Se realizaron el test de Kruskal-Wallis seguido por el test de comparaciones multiples de Dunn y test t seguido por el test de Mann Whitney (twotailed) (GraphPad Prism v 6.00). Valores de $p<0,05$ con respecto a LPS fueron considerados significativos. BCII=pacientes con cáncer de mama estadio II; $\mathrm{H}=$
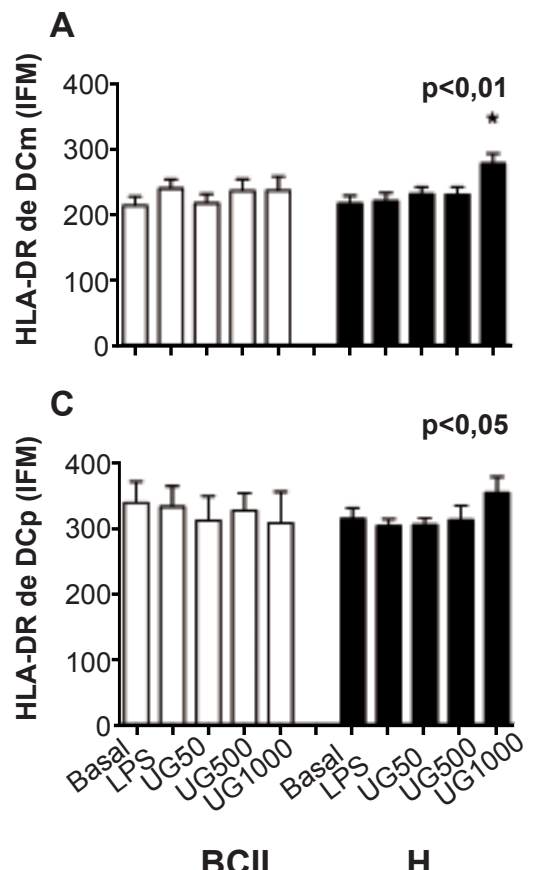

BCII
B

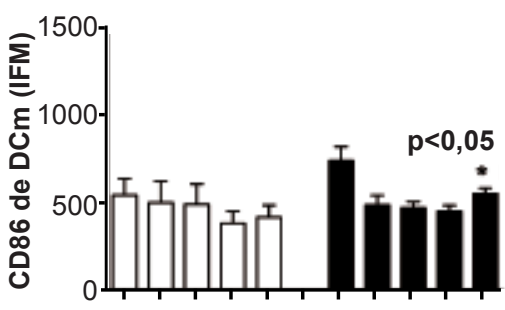

D

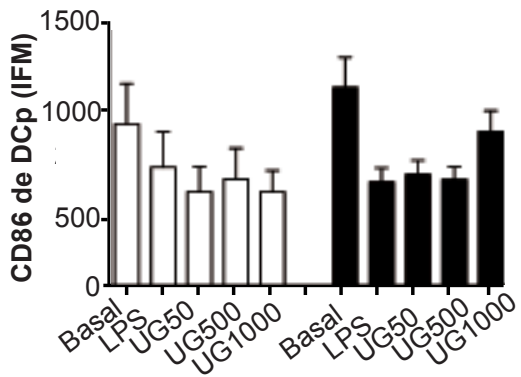

BCII

mujeres sanas con mamografías negativas; UT-POA= extracto hidroalcohólico de Uncaria tomentosa con 5,03\% de alcaloides oxindólicos pentacíclicos; HLA-DR = Antígeno Leucocitario Humano, haplotipo DR o complejo mayor de histocompatibilidad tipo II; CD86= mólecula coestimuladora; IFM= intensidad de fluorescencia media, $\mathrm{PBMC}=$ células mononucleares de sangre periférica, ${ }^{*} \mathrm{p}<0,05$. 
(Figura 1). El grupo BCll demostró una disminución significativa del \%DCm a $1000 \mu \mathrm{g} / \mathrm{mL}(p<0,05)$ (Figura 1A). UT-POA disminuyó significativamente el \%DCp a $1000 \mu \mathrm{g} / \mathrm{mL}(p<0,05)$ en el grupo H (Figura 1B).

La molécula HLA-DR de DCm y DCp se incrementó significativamente $(p<0,05)$ en el grupo $\mathrm{H}$ a $1000 \mu \mathrm{g} / \mathrm{mL}$; mientras que en el grupo BCII no se encontró variación (Figura $2 \mathrm{~A}$ y $2 \mathrm{C}$ ). Nínguna diferencia fue observada para CD86 (Figura 2B y 2D).
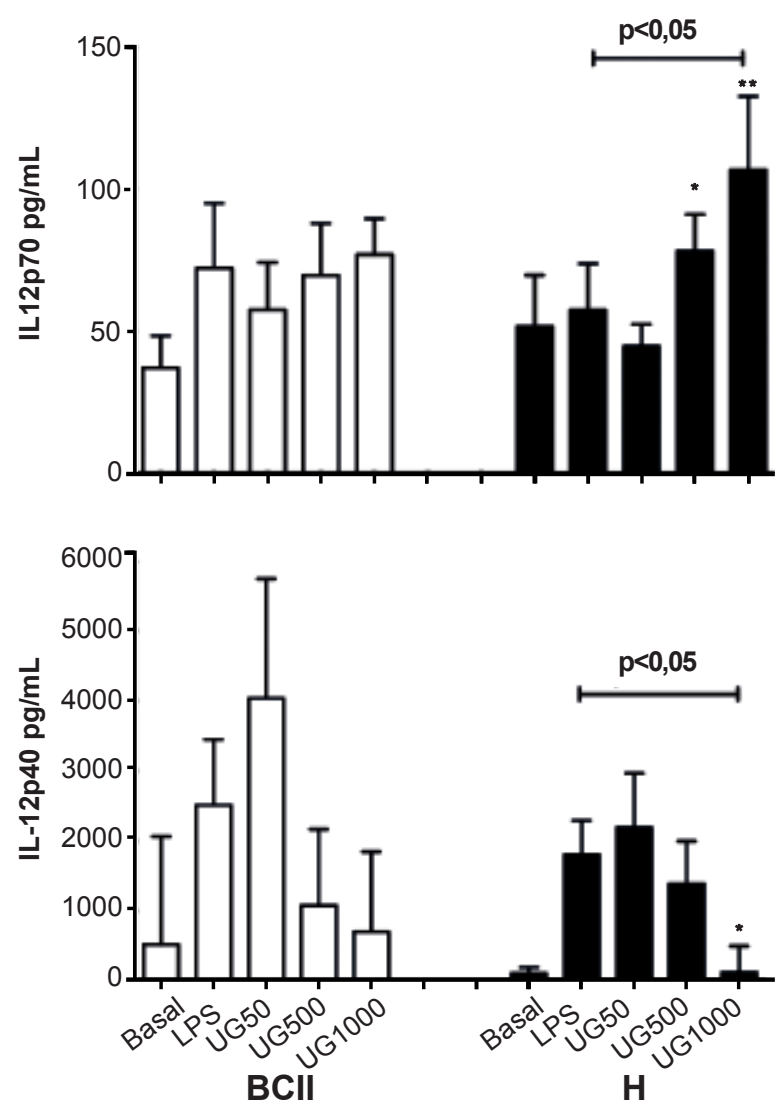

Figura 3. Medición de los niveles de IL-12p70y lL-12p40 (pg/mL). Después del aislamiento y cultivo de PBMC para la citometría de flujo, se colectaron los sobrenadantes de cultivo para medir las dos isoformas de IL-12 utilizando la técnica de ELISA. A). Niveles incrementados de IL-12p70 que fueron significativos para el grupo $\mathrm{H}$ a $1000 \mathrm{ug} / \mathrm{mL}$. B). Niveles disminuidos de IL12p40 que fueron significativos para el grupo $\mathrm{H}$ a $1000 \mathrm{ug} / \mathrm{mL}$. Los datos representan el promedio \pm error estándar de la media de $7 \mathrm{BCIl}$ y $10 \mathrm{H}$. Se realizo el test no paramétrico de KruskalWallis seguido por el test de comparaciones multiples de Dunn. Valores de $p<0,05$ con respecto a LPS fueron considerados significativos. $\mathrm{BCl}=$ pacientes con cáncer de mama estadio II; $\mathrm{H}=$ mujeres sanas con mamografías negativas; $\mathrm{PBMC}=$ células mononucleares de sangre periférica; ${ }^{*} p<0,05 ;{ }^{* *} p<0,01$.

\section{IL-12p70 y IL-12p40}

IL-12p70 se incrementó significativamente en el grupo BCll a $1000 \mu \mathrm{g} / \mathrm{mL}(p<0,05)$; sin embargo, en el grupo $\mathrm{H}$ se incrementó de forma dosis-dependiente siendo significativo a 500 y $1000 \mu \mathrm{g} / \mathrm{mL}(p<0,05)$ (Figura $3 A)$. IL-12p40 en ambos grupos mostró una disminución dosis-dependiente significativa solo para el grupo $\mathrm{H}$ a $1000 \mu \mathrm{g} / \mathrm{mL}$ comparado con LPS $(p<0,05)$ (Figura 3B).

\section{CITOQUINAS Th1}

En BCIl y H, los niveles de IFN-k y IL-2 se incrementaron de manera dosis-dependiente a 500 y $1000 \mu \mathrm{g} / \mathrm{mL}$ $(p<0,05)$ comparado con LPS. Se observaron mayores niveles de IFN-k y IL-2 en el grupo H que en BCII (Figura $4 \mathrm{~A}$ y $4 \mathrm{~B}$ ). Los niveles de TNF- $\alpha$ no cambiaron en ningún grupo (Figura 4C).

\section{CITOQUINAS Th2}

En $\mathrm{BCll}$ y $\mathrm{H}$, hubo un incremento dosis-dependiente de IL-4 $(p<0,05)$ a 500 y $1000 \mu \mathrm{g} / \mathrm{mL}$ comparado con LPS. También se observó que el grupo $\mathrm{H}$ producía más IL-4 que el grupo BCII (Figura 4D). IL-6 y IL-10 no cambiaron (Figura 4E y Figura 4F). Sin embargo, en el grupo H IL-6 disminuyó significativamente a $50 \mu \mathrm{g} / \mathrm{mL}$ que luego se mantuvo al mismo nivel que el grupo LPS (Fig 4F). Hubo una tendencia dosis-dependiente a incrementar IL-10 observándose mayor cantidad de esta citoquina que en el grupo BCII (Figura 4E).

\section{CITOQUINAS TH17}

Existió un incremento dosis-dependiente de IL-17A en los grupos $\mathrm{BCll}$ y $\mathrm{H}$ que se hizó estadíticamente significativo a $1000 \mu \mathrm{g} / \mathrm{mL}(p<0,05)$ (Figura 4G).

\section{DISCUSIÓN}

La UT es un fitoquímico inmunomodulador, antinflamatorio y antioxidante. Estudios previos correlacionaron el contenido de alcaloides oxindólicos pentacíclicos (POA) de sus hojas, cortezas y raices con las propiedades inmunomoduladoras de esta especie (22). Varios fitoquímicos preparados a partir de extractos etanólicos de corteza y la fracción rica en alcaloides de UT han sido estandarizados por sus contenidos de alcaloides oxindólicos ${ }^{(1,23)}$; sin embargo, esto no significa que los POA sean los causantes de los efectos de la UT, porque existen productos como C-Med $100 \AA$, un extracto patentado de UT soluble en agua y ultrafiltrado para remover conjugados de alto peso molecular $(>10 \mathrm{kDa})$ que contiene ésteres de carboxylalquil (CAE) como ingredientes activos $(8-10 \%)$ y casi 


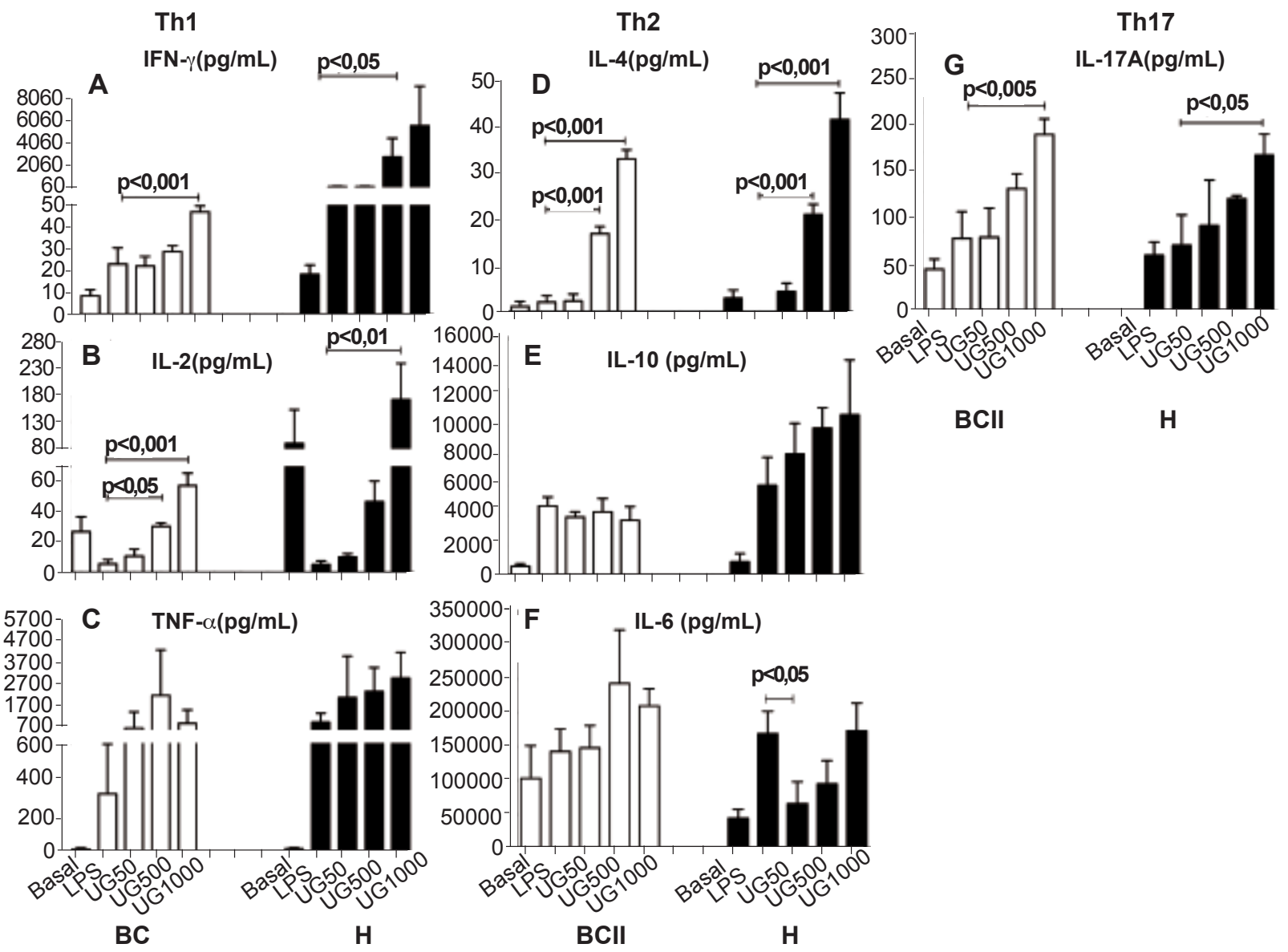

Figura 4. Medición de los niveles de las citoquinas Th1/Th2/Th17. Después del aislamiento y cultivo de PBMC para la citometría de flujo, se colectaron los sobrenadantes de cultivo para medir las citoquinas Th1/Th2/Th17 por CBA. A) IFN-g; B) IL-2; C) TNF- $\alpha$; D) IL-4; E) IL-10 y F) IL-6. Los datos representan el promedio \pm error estándar de la media de 7 BCll y $10 \mathrm{H}$. Se realizaron el test de Kruskal-Wallis seguido por el test de comparaciones multiples de Dunn y test t seguido por el test de Mann Whitney (two-tailed) (GraphPad Prism v 6.00) Valores de $p<0,05$ con respecto a LPS fueron considerados significativos. BCll=pacientes con cáncer de mama estadio II; $\mathrm{H}=$ mujeres sanas con mamografías negativas: $\mathrm{CBA}=$ Cytometric Bead Array; $\mathrm{PBMC}=$ células mononucleares de sangre periférica; ${ }^{*} p<0,05 ;{ }^{* *} p<0,01 ;{ }^{* *} p<0,001$.

libre de alcaloides oxindólicos $(\leq 0,05 \%)$ que presenta efectos inmunomoduladores ${ }^{(24)}$. Por lo tanto, es posible que varios ingredientes activos de la planta actuen en sinergía.

Investigamos si la UT era capaz de modificar el inmunofenotipo de DC e inmunomodular las citoquinas IL-12, Th1, Th2 y Th17 in vitro en PBMC humanos. Encontramos que UT-POA tiene un efecto inmunomodulador aparentemente opuesto sobre las DC y su marcador HLA-DR, ya que en el primer caso disminuye los marcadores específicos de estas células y en el segundo caso incrementa la expresión de la molécula relacionada con la maduración (HLA-DR); mientras que no se ha encontrado que logre inducir la activación de estas células al no tener efecto sobre la molécula coestimuladora CD86. No existen muchos estudios que evaluen el efecto de la UT sobre DC humanas; Kim KS et al. ensayaron otras especies como Uncaria rhynchophylla y especificamente el ácido uncarinico (URC) y encontraron que las DC aisladas de $P B M C$ de humanos sanos aumentaron la expresión de CD1A, CD38, CD40, CD54, CD80, CD83, CD86, HLA-DR y DC-Lamp después del tratamiento con URC en el rango de 0,001 a $10 \mu \mathrm{M}{ }^{(25)}$. En el caso de UT, Nunez C, et al. y Lozada-Requena et al. demostraron que el \%DCm en PBMC de pacientes con artritis reumatoide y en sujetos sanos, disminuyó de manera dosis dependiente, mientras que HLA-DR y CD86 se incrementaron en pacientes con artritis, pero no se modificaron en sujetos sanos ${ }^{(18,26)}$. Confirmamos los hallazgos de Lozada-Requena I et al., con relación a la disminución del \%DCm del grupo $\mathrm{H}$ y, además, encontramos este mismo resultado en el grupo BCII; sin embargo, en el caso del \%DCp nuestros resultados son opuestos pues se encontró más bien una disminución 
de esta subpoblación en el grupo $\mathrm{H}$. Es posible que la enfermedad de estas pacientes tuviera un efecto protector/bloqueador contra UT-POA actuando con mecanismos de evasión típicos, inducidos por tumores y producidos por factores liberados por el tumor o algún otro mecanismo que desconocemos. Por lo tanto, no pudimos encontrar algún efecto inmunomodulador de UT-POA sobre las DCp y HLA-DR/CD86 de pacientes con cáncer de mama, excepto la disminución de las $\mathrm{DCm}$ a concentraciónes altas. La disminución de esta subpoblación de $\mathrm{DCm}$ a nivel sistémico no necesariamente se debe considerar un mal pronóstico ya que se debe tener en cuenta que las DC representan entre el $0,1-1 \%$ de las poblaciones inmunocompetentes sanguíneas y que es posible su migración al microambiente tumoral. Sugerimos estudios adicionales con extractos estandarizados a dosis diferentes y en el microambiente tumoral.

La producción de IL-12p70 fue aumentada e IL-12p40 disminuyó. IL-12p70 es responsable de la polarización Th1 mediada por DC. Nuestros hallazgos demuestran que los PBMC estimulados in vitro con UT-POA incrementan las citoquinas Th1 (IFN $\square \mathrm{k}$ y IL $\square$ 2), Th2 (IL4) y Th17 (IL-17) en BCII y H. Aunque estos efectos se observan a dosis altas debemos tener en cuenta que el valor de IC50 de UT enfrentada con PBMC humanos es de $16 \mathrm{mg} / \mathrm{mL}$, lo que indica que UT no afecta la viabilidad celular de los PBMC ${ }^{(18)}$, sugerimos realizar mediciones sobre la viabilidad metabólica de estas células frente a UT; también deberíamos considerar que durante la preparación de UT existen pasos de filtración que podrían diluir el extracto, por lo que las concentraciones utilizadas deben ser tomadas como referenciales.

Demostramos que ni IL-12p70 ni la subunidad IL12p40 son modificados por UT-POA en el grupo BCII. Sin embargo, en el grupo $\mathrm{H}, \mathrm{IL}-12 \mathrm{p} 70$ se incrementó y IL-12p40 disminuyó. La explicación se debería a que parte de IL-12p40 se dimerizaría con la IL-12p35 para formar IL-12p70. Es bien sabido que la familia de IL12 esta formada por IL-12, IL-23 y IL-27 y que la IL12 p40 no solo genera IL-12 sino que también genera IL-23 uniéndose a IL-12p19, por lo tanto es posible que parte de IL-12p40, la cual disminuyó en el grupo $\mathrm{H}$, se haya dimerizado con la subunidad IL-12p19 (27,28); sin embargo, esta hipótesis debe ser demostrada midiendo IL-23. De acuerdo con nuestros hallazgos, al menos en sujetos sanos, UT-POA favorece la producción de IL-12. Las pacientes del grupo BCll presentarían un desorden en sus PBMC que inhibiría una respuesta favorable al extracto de UT-POA. La razón de esta falta de respuesta es la misma que proponemos en relación a las DC, es decir, la presencia de evasion tumoral u otros mecanismos que desconocemos.
Sobre el perfil Th1/Th2/Th17, encontramos que UT-POA incrementó las Th1 (IFN-k y IL-2), Th2 (IL-4) y Th17 (IL$17 \mathrm{~A}$ ) en los grupos $\mathrm{BCIl}$ y $\mathrm{H}$. Existen pocos estudios acerca del efecto de la UT sobre las citoquinas Th1, Th2 y Th17; sin embargo, Domingues $A$, et al. encontraron que los esplenocitos de ratones tratados con un extracto hidroalcohólico de uña de gato presentaban un perfil Th2 caracterizado por un incremento de IL-4 y IL-5 a $500 \mu \mathrm{g} / \mathrm{mL}$, mientras que a la misma concentración se producia una disminución de las citoquinas Th1 (IFN-k, IL-2 y TNF- $\alpha){ }^{(19)}$. Fazio $A L$ et al. encontraron que las Th1 (TNF-k) y Th2 (IL-6) estaban disminuidas en el sobrenadante de cultivos de macrófagos peritoneales tratados con UT, luego de la estimulación con LPS, y demostró que esta disminución se correlacionaba con una imperfección de la vía NF-kB ${ }^{(20)}$. Aunque estos estudios fueron realizados en modelos animales, demostramos que la uña de gato puede tener diferentes efectos moduladores sobre la expresión de diferentes citoquinas y que los resultados no necesariamente se deben extrapolar del ratón al humano. Demostramos una inducción de la respuesta Th1 en el grupo $\mathrm{H}$, probablemente por el incremento de IL-12p70 y, por lo tanto, justificaría el incremento de IFN-k y IL-2; sin embargo, en el grupo BCll es posible que UT-POA directamente estimule el incremento de IFN-k y IL-2 por mecanismos que deben ser investigados. Ambas citoquinas son importantes para la respuesta inmune, mientras que IL-2 funciona como un factor de crecimiento necesario para la supervivencia y diferenciación de LT y es producida principalmente por LT CD4, lo que explica porque su presencia e incremento podría mejorar la respuesta inmune adaptativa antitumoral, el IFN-k tiene un rol directo en la inmunidad antitumoral; no solo en la inmunidad adaptativa, sino también en la innata, ya que puede tener distintos efectos sobre componentes endoteliales y estromales en el microambiente tumoral, lo cual puede inducir quimioquinas que atraen células efectoras desde ambos brazos del sistema inmune e inhibir la angiogenesis y moléculas adherentes de las células endoteliales.

En relación a TNF-a, se conoce un efecto inhibidor de UT sobre su producción, diferentes estudios han demostrado un efecto antinflamatorio en una variedad de modelos in vitro e in vivo. Los resultados no demuestran variación de TNF-a en los grupos ensayados. Es posible que mecanismos que desconocemos impidan su inhibición o que el tiempo de tratamiento con UT-POA y la estimulación con LPS no hayan sido suficientes y deban estandarizarse para este modelo en particular. Además, los otros estudios se hiceron con líneas celulares estandarizadas o líneas celulares específicas como macrófagos peritoneales, mientras que nosotros utilizamos PBMC, los que involucran a otros leucocitos que podrían inducir mayor variabilidad en las mediciones. 
En ambos grupos, UT-POA incrementó IL-4. La fuente de IL-4 son los LT, mastocitos y otras poblaciones celulares y es requerida para la diferenciación Th2. El uso de PBMC implica que la principal fuente de IL-4 sean los LT. Aunque se esperaría un perfil disminuido de Th2 debido a un prevalente perfil Th1, UT-POA también incrementó el perfil Th2 (IL-4), demostrando una vez más su efecto inmunomodulador. A pesar de la renuencia de IL-4 en la inmunidad antitumoral, porque su presencia induciría la polarización de macrófagos M2 o TAM (macrófagos asociados al tumor) que son más bien protumorales, IL-4 colaboraría con linfocitos B productores de $\lg G$ y $\lg E$ e incrementaría la expresión de MHC, mejorando también la activación de LT y la función de los eosinófilos ${ }^{(8)}$.

Algunos anticuerpos dirigidos contra móleculas presentes en células cancerígenas pueden inhibir señales oncogénicas y promover la destrucción tumoral a través de la unión a receptores Fc de macrófagos, granulocitos y células NK. Los anticuerpos también pueden promover la presentación de antígenos tumorales por CPA por la formación de complejos inmunes ${ }^{(8)}$. Por estas razones, la diferenciación Th2 (IL-4) no debería ser del todo descartada en la respuesta antitumoral.

Demostramos que IL-10 no fue afectada por UT-POA en $\mathrm{BCll} \circ \mathrm{H}$; sin embargo, la producción de IL-6 fue disminuida a $50 \mu \mathrm{g} / \mathrm{mL}$ inicialmente y luego de manera dosis-dependiente alcanzó los niveles del grupo LPS. Aunque Lemaire et al. demostraron un efecto inhibidor de dos extractos acuosos de UT sobre IL-6 en macrófagos alveolares de ratas (29); aquí demostramos que UT-POA puede tener diferentes efectos a distintas concentraciones denotando la importancia de determinar la concentración óptima a la que se dan estos efectos.

Los linfocitos Th17 han sido recientemente descritos en humanos y se les atribuye un rol en la inflamación de enfermedades autoinmunes; sin embargo, también son críticos en la respuesta antitumoral porque inducen la producción de quimioquinas por los tumores primarios, las que atraen a DC, LT y células NK hacia el microambiente tumoral ${ }^{(30)}$. Sin embargo, también existen evidencias de la correlación entre
LT reguladores y LTh17 de pacientes con cáncer de mama invasivo, sugiriendo que el incremento de estas subpoblaciones resulta en condiciones que favorecen la agresividad del tumor y pobres pronósticos de la enfermedad. En otros tumores como el de ovario, las Th17 inducen quimioquinas tipo Th1 y el reclutamiento de células efectoras. Así, las Th17 y los LT reguladores tendrían un rol dual en el microambiente tumoral (31). Estas aparentes discordancias de las Th17 y la IL17-A deberían ser elucidadas muy pronto. En la actualidad no existen estudios que evaluen el efecto de UT-POA sobre la expresión de IL-17. Nuestros resultados demuestran que en los grupos $\mathrm{H}$ y $\mathrm{BCll}$ hubo un incremento dosisdependiente de la producción de IL-17A. La producción de IL-17A fue siempre mayor en el grupo $\mathrm{H}$ que en $\mathrm{BCII}$ demostrando que aunque el cáncer de mama afecta la secreción de IL-17A, estos niveles son recuperados por UT-POA.

Los datos obtenidos en los ensayos con PBMC de pacientes con cáncer de mama sugieren un importante efecto inmunomodulador de la uña de gato sobre la expresión de citoquinas Th1 (IFN-g y IL-2), Th2 (IL-4) y Th17 (IL-17A), lo que requiere más investigaciones que confirmen la actividad citoprotectora de este fitoquímico en la respuesta antitumoral.

Agradecimientos: este trabajo ha sido subvencionado por EMINDES CANCER SAC (Empresa de Investigación y Desarrollo en Cáncer). El servicio de citometría de flujo ha sido realizado por el Laboratorio de Inmunología (LID-108), Laboratorios de Investigación y Desarrollo (LID), Universidad Peruana Cayetano Heredia, Lima-Perú.

Contribuciones de autoría: CN y ILR participaron en la concepción y diseño del protocolo del estudio y en la obtención del financiamiento. ILR, TY participaron en la recolección de muestras, obtención de resultados y realización del análisis estadístico de los datos. CN, ILR, TY, DZ, FS y JA participaron en el análisis y redacción del artículo. Todos los autores han dado su revisión crítica del artículo.

Fuentes de financiamiento: este estudio fue financiado por EMINDES SAC.

Conflictos de interés: No existe ningún potencial conflicto de interés.

\section{REFERENCIAS BIBLIOGRÁFICAS}

1. Keplinger K, Laus G, Wurm M, Dierich MP, Teppner H. Uncaria tomentosa (Willd.) DC.- - ethnomedicinal use and new pharmacological, toxicological and botanical results. J Ethnopharmacol. 1999;64(1):23-34.

2. Aquino R, De Feo V, De Simone F, Pizza C, Cirino G. Plant metabolites. New compounds and anti-inflammatory activity of Uncaria tomentosa. J Nat Prod. 1991;54(2):453-9.

3. Pilarski R, Poczekaj-Kostrzewska M, Ciesiolka D, Szyfter K, Gulewicz K. Antiproliferative activity of various Uncaria tomentosa preparations on HL-60 promyelocytic leukemia cells. Pharmacol Rep. 2007; 59(5):565-72.
4. Sandoval-Chacón M, Thompson $\mathrm{JH}$, Zhang XJ, Liu X, Mannick EE, Sadowska-Krowicka $\mathrm{H}$, et al. Antiinflammatory actions of cat's claw: the role of NF-kappaB. Aliment Pharmacol Ther. 1998; 12(12):1279-89.

5. Aguilar JL, Rojas P, Marcelo A, Plaza A, Bauer R, Reininger E, et al. Anti-inflammatory activity of two 
different extracts of Uncaria tomentosa (Rubiaceae). J Ethnopharmacol 2002; 81(2):271-6.

6. Dreifuss AA, Bastos-Pereira AL, Ávila TV, Soley Bda S, Rivero AJ, Aguilar $\mathrm{JL}$, et al. Antitumoral and antioxidant effects of a hydroalcoholic extract of cat's claw (Uncaria tomentosa) (Willd. Ex Roem. \& Schult) in an in vivo carcinosarcoma model. J Ethnopharmacol. 2010; 130(1):12733. doi: 10.1016/j.jep.2010.04.029.

7. Dreifuss AA, Bastos-Pereira AL, Fabossi IA, Livero FA, Stolf AM, Alves de Souza CE, et al. Uncaria tomentosa exerts extensive anti-neoplastic effects against the Walker-256 tumour by modulating oxidative stress and not by alkaloid activity. PLoS One. 2013; 8(2):e54618. doi:10.1371/journal. pone. 0054618 .

8. Dranoff G. Cytokines in cancer pathogenesis and cancer therapy. Nat Rev Cancer. 2004;4(1):11-22.

9. de Visser KE, Eichten A, Coussens LM. Paradoxical roles of the immune system during cancer development. Nat Rev Cancer. 2006;6(1):24-37.

10. Karin M. NF-kappaB as a critical cink between inflammation and cancer. Cold Spring Harb Perspect Biol. 2009; 1(15):a000141. doi: 10.1101/ cshperspect.a000141.

11. Allen-Hall L, Arnasond JT, Cano P, Lafrenie RM. Uncaria tomentosa acts as a potent TNF-alpha inhibitor through NF-kappaB. J Ethnopharmacol. 2010;127(3):68593. doi: 10.1016/j.jep.2009.12.004.

12. Fuertes MB, Zwirner NW. Estrategias celulares de defensa contra cáncer. In: Aguilar JL, editor. Bases de la Inmunología Clínica. $1^{\text {ra }}$ ed. Lima: Sociedad Peruana de Inmunología (SPI); 2013. p.147-68.

13. Steinman RM, HawigerD, Nussenzweig MC. Tolerogenic dendritic cells. Annu Rev Immunol. 2003;21:685-711.

14. Pulendran B, Smith JL, Caspary G, Brasel K, Pettit D, Maraskovsky E, et al. Distinct dendritic cell subsets differentially regulate the class of immune response in vivo. Proc Natl Acad Sci USA. 1999;96(3):1036-41.

15. Almand B, Resser JR, Lindman B, Nadaf S, Clark JI, Kwon $\mathrm{ED}$, et al. Clinical significance of defective dendritic cell differ- entiation in cancer. Clin Cancer Res. 2000;6(5):1755-66.

16. Esche C, Lokshin A, Shurin GV, Gastman BR, Rabinowich H, Watkins $\mathrm{SC}$, et al. Tumor's other immune targets: dendritic cells. J Leukoc Biol. 1999; 66(2):336-344.

17. Tas M, Simons P, Balm F, and Drexhage H. Depressed monocyte polarization and clustering of dendritic cells in patients with head and neck cancer: in vitro restoration of this immunosuppression by thymic hormones. Cancer Immunol Immunother. 1993;36(2):108-14.

18. Núñez C, Lozada-Requena I, Akamine I, Carbajal L, Aguilar JL. Efecto de Uncaria tomentosa (Uña de Gato) sobre la población y activación de células dendríticas de sangre periférica de pacientes con artritis reumatoidea. Acta Med Per. 2008;25(3):135-9.

19. Domingues A, Sartori A, Valente LM, Golim MA, Siani AC, Viero RM. Uncaria tomentosa aqueous-ethanol extract triggers an immunomodulation toward a Th2 cytokine profile. Phytother Res. 2011;25(8):1229-35. doi: 10.1002/ptr.3549.

20. Fazio AL, Ballen D, Cesari IM, Abad MJ, Arsenak M, Taylor P. An ethanolic extract of Uncaria tomentosa reduces inflammation and B16-BL6 melanoma growth in C57BL/6 mice. Bol Latinoam Caribe Plant Med Aromaticas. 2008; 7(4):217-224.

21. Urdanibia I, Michelangeli F, Ruiz MC, Milano B, Taylor P. Anti-inflammatory and antitumoural effects of Uncaria guianensis bark. J Ethnopharmacol. 2013;150(3):1154-62. doi: 10.1016/j.jep.2013.10.055.

22. Winkler C, Wirleitner B, Schroecksnadel K, Schennach $\mathrm{H}$, Mur E, Fuchs D. In vitro Effects of Two Extracts and Two Pure Alkaloid Preparations of Uncaria tomentosa on Peripheral Blood Mononuclear Cells. Planta Med. 2004; 70(3):205-10.

23. Keplinger $\mathrm{K}$, Wagner $\mathrm{H}$, Kreutzkamp B. Oxindole alkaloids having properties stimulating the immunologic systems. Washington DC: United States Patent $\mathrm{N}^{\circ}$ 4844901, 1989.

24. Åkesson Ch, Pero RW, Ivars F. C-Med 100, a hot water extract of Uncaria tomentosa, prolongs lymphocyte survival in vivo. Phytomedicine 2003;10(1):23-33.

25. Kim KS, Pham TNN, Jin Ch-J, Umeyama A, Shoji N, Hashimoto $\mathrm{T}$, et al. Uncarinic acid $\mathrm{C}$ isolated from Uncaria rhynchoplylla induce differentiation of Th1-promoting dendritic cells through TLR 4 signaling. Biomarker Insights. 2011;6:27-38. doi: 10.4137/BMI.S6441.

26. Lozada-Requena, I, Núñez C, Álvarez Y, Aguilar JL. Efecto de un extracto hidroalacohólico de Uncaria tomentosa (uña de gato) sobre la población de células dendríticas y sus moléculas HLA-DR y CD86 ante el estímulo con lipopolisacáridos. Rev Peru Med Exp Salud Publica. 2009; 26(2):168-74.

27. Hunter CA. New IL-12 family members: IL-23 and IL-27, cytokines with divergent functions. Nat Rev Immunol. $2005 \mathrm{Jul} ; 5(7): 521-31$.

28. Xu M, Mizoguchi I, Morishima N, Chiba Y, Mizoguchi J, Yoshimoto T. Regulation of antitumor immune response by the IL-12 family cytokines, IL-12, IL-23, and IL-27. Clin Dev Immunol. 2010;2010. pii: 832454. doi: $10.1155 / 2010 / 832454$.

29. Lemaire I, Assinewe V, Cano P, Awang DVC, Arnason JT. Stimulation of interleukin-1 and -6 production in alveolar macrophages by the neotropical liana, Uncaria tomentosa (Uña de Gato). J Ethnopharmacol 1999; 64(2):109-115

30. Zou W, Restifo NP. T(H)17 cells in tumour immunity and immunotherapy. Nat Rev Immunol. 2010 Apr;10(4):248-56. doi: 10.1038/ nri2742.

31. Benevides L, Cardoso CR, Tiezzi DG, Marana HRC, Andrade JM, Silva JS. Enrichment of regulatory $\mathrm{T}$ cells in invasive breast tumor correlates with the upregulation of IL-17A expression and invasiveness of the tumor. Eur J Immunol. 2013; 43(6):15181528. doi: 10.1002/eji.201242951.

Correspondencia: Ivan Lozada-Requena. Direccion: Av. Honorio Delgado 430, San Martin de Porres, Lima 31, Perú.

Telefono: +51-319-0000 anexo 2511. Correoelectrónico:ivan.lozada@upch.pe 